\title{
Aggression and social experience: genetic analysis of visual circuit activity in the control of aggressiveness in Drosophila
}

\author{
Mahmoudreza Ramin ${ }^{1,2}$, Claudiu Domocos ${ }^{1}$, David Slawaska-Eng ${ }^{1}$ and Yong Rao 1,2,3*
}

\begin{abstract}
Background: Animal aggressiveness is controlled by genetic and environmental factors. Among environmental factors, social experience plays an important role in modulating aggression in vertebrates and invertebrates. In Drosophila, pheromonal activation of olfactory neurons contributes to social suppression of aggression. While it was reported that impairment in vision decreases the level of aggression in Drosophila, it remains unknown if visual perception also contributes to the modulation of aggression by social experience.

Results: In this study, we investigate the role of visual perception in the control of aggression in Drosophila. We took several genetic approaches to examine the effects of blocking visual circuit activity on fly aggressive behaviors. In wild type, group housing greatly suppresses aggressiveness. Loss of vision by mutating the ninaB gene does not affect social suppression of fly aggression. Similar suppression of aggressiveness by group housing is observed in fly mutants carrying a mutation in the eya gene leading to complete loss of eye. Chronic visual loss does not affect the level of aggressiveness of single-housed flies that lack social experience prior to behavioral tests. When visual circuit activity is acutely blocked during behavioral test, however, single-housed flies display higher levels of aggressiveness than that of control flies.
\end{abstract}

Conclusion: Visual perception does not play a major role in social suppression of aggression in Drosophila. For single-housed individuals lacking social experience prior to behavioral tests, visual perception decreases the level of aggressiveness.

\section{Background}

Aggression is an innate behavior that allows animals to compete for limited resources, such as food, mating partners and habitats. The level of aggressiveness is influenced by both genetic and environmental factors [1]. Accumulated evidence supports that social experience is one of the most important environmental factors that affect aggression in humans [2], rats [3-5] and Drosophila [6].

Recent studies have shed light on molecular mechanisms underlying the control of aggression by social experience. For instance, Cyp6a20, a cytochrome P450, is identified as a common genetic target for the control of

\footnotetext{
* Correspondence: yong.rao@mcgill.ca

'McGill Centre for Research in Neuroscience, McGill University Health Centre, 1650 Cedar Avenue, Montreal, Quebec H3G 1A4, Canada

${ }^{2}$ Department of Neurology and Neurosurgery, Integrated Program in

Neuroscience, McGill University Health Centre, 1650 Cedar Avenue, Montreal, Quebec H3G 1A4, Canada

Full list of author information is available at the end of the article
}

aggressiveness by social experience in Drosophila [7]. It has also been reported that chronic activation of Or65a olfactory neurons by the volatile pheromone 11cis-vaccenyl acetate (cVA) contributes to social suppression of aggressiveness in Drosophila [8,9]. However, it remains unknown if other sensory stimuli such as vision, also contributes to social suppression of aggression in Drosophila.

A previous study reports that mutations in the white (w) gene that regulates eye pigmentation, greatly decrease aggressiveness of single-housed flies, suggesting that vision is required for normal aggression [10]. To determine if visual perception contributes to social suppression of aggressiveness, we investigated if the blockade of visual circuit activity affects social suppression of aggression. We also examined the effects of visual impairment on aggressiveness of single-housed flies that lack social experience prior to behavioral tests. 

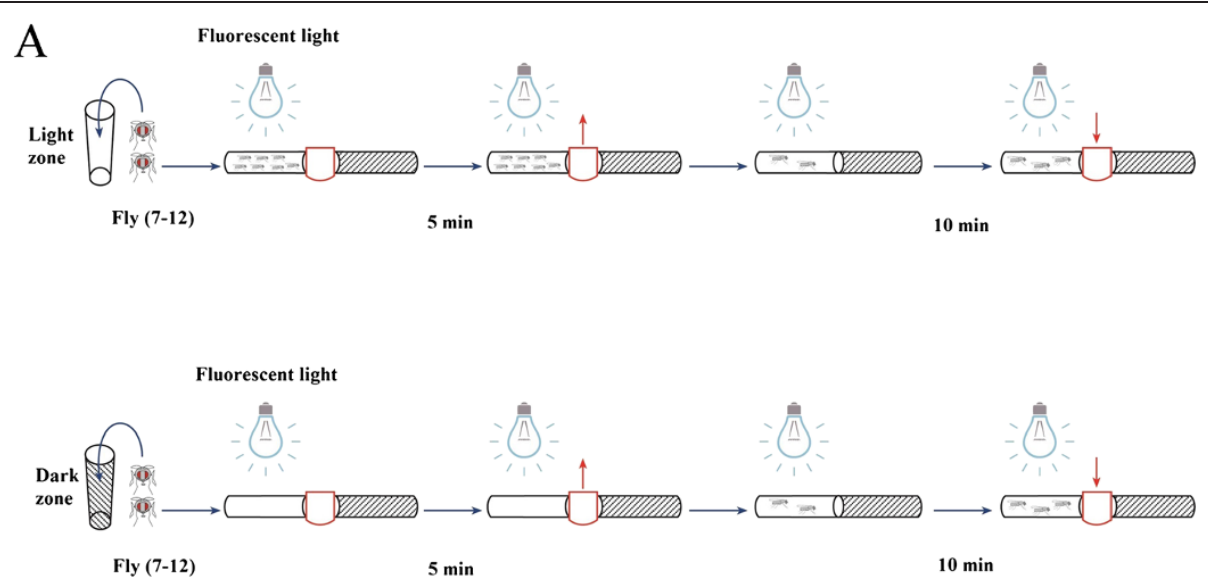

B

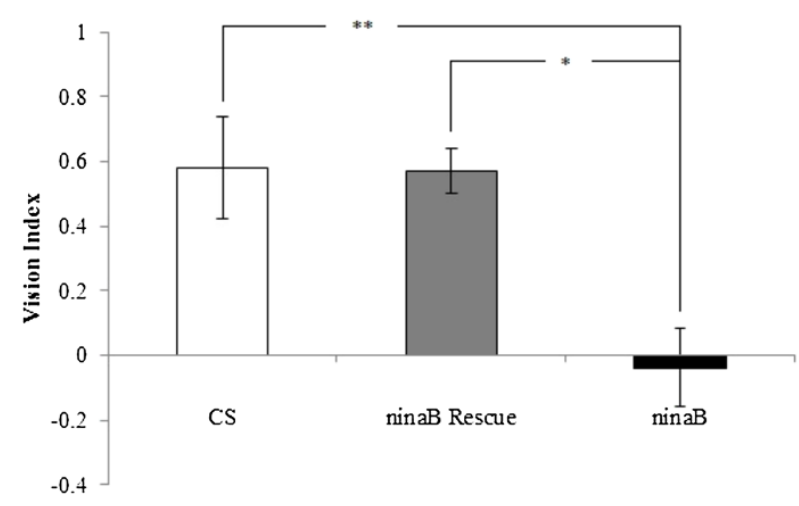

C

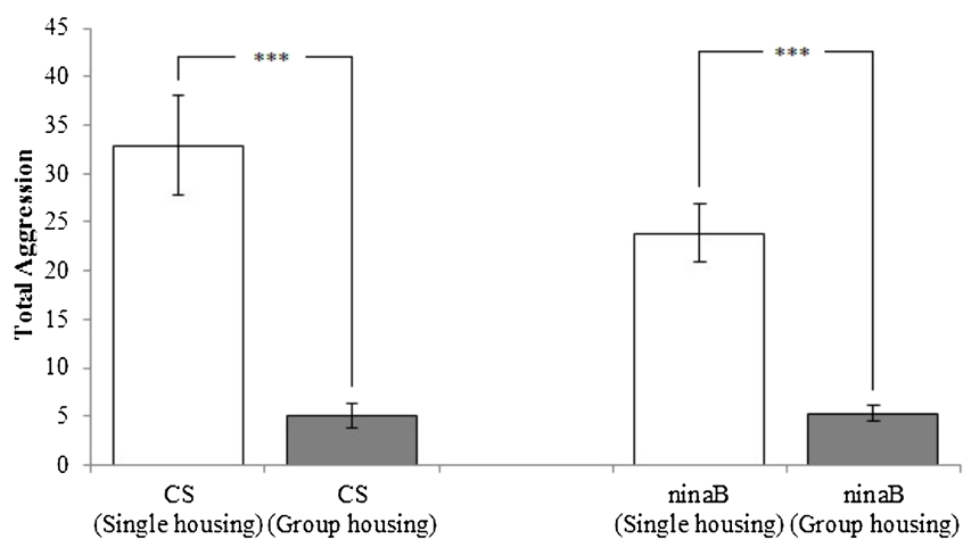

Figure 1 Visual impairment in ninaB mutants does not affect social suppression of aggressiveness. (A) Schematic drawing of phototaxis assay (see Materials and methods). (B) Vision index of flies was quantified (see Materials and methods). Canton-S (CS) wild-type flies prefer to stay in light zone. Whereas ninaB ${ }^{1}$ mutant flies distributed randomly in light and dark zones, indicating impairment in vision. Rescued flies in which a $U A S-n i n a B$ transgene was expressed in photoreceptors in ninaB ${ }^{1}$ mutant flies under control of the eye-specific GMR-GAL4 driver, showed light preference similar to that of wild-type flies. ${ }^{* *} p<0.01,{ }^{*} p<0.05$. Number of experiments performed: $C S, n=11$; nina $B^{1}$ Rescue, $n=10 ; n i n a B^{1}$, $n=11$. (C) Social suppression of aggressiveness of wild-type and nina $B^{1}$ mutant flies. The level of aggressiveness (i.e. total aggression) was quantified by counting the number of all aggressive events (i.e. lunges, wing threats, tussles, boxing, and holding) within 10-min period. Pairs of flies tested: $C S, n=27$ (single housing), $n=21$ (group housing); ninaB ${ }^{1}$ mutants, $n=22$ (single housing), $n=20$ (group housing). ${ }^{* * *} p<0.0001$. Error bars represent SEM. 


\section{Results}

Loss of vision in nina $B$ mutants does not prevent social suppression of aggression

To determine if visual perception contributes to social suppression of fly aggression, we examined if the modulation of aggressiveness by social experience is affected in blind nina $B$ mutant flies. ninaB encodes a $\beta, \beta$-carotene$15,15^{\prime}$-dioxygenase that mediates the generation of visual chromophores [11]. To confirm that ninaB ${ }^{1}$ mutation causes loss of vision [12], we performed phototaxis experiments similarly as described previously [13]. For each experiment, $\sim 7-12$ flies were aspirated into dark or light zone, and then allowed to move freely (Figure 1A). Wildtype flies or rescue flies in which a ninaB transgene was expressed in nina $B^{1}$ mutants show a preference for light zone (Figure 1B). By contrast, ninaB ${ }^{1}$ mutants selected light or dark zone randomly. This result confirms that vision is impaired in nina $B^{1}$ mutants.

We then performed experiments to examine the level of aggressiveness in flies with or without social experience. Wild-type flies reared in isolation (single housing) displays a much higher level of aggressiveness compared to flies reared in group (group housing) (Figure 1C), indicating that social experience prior to aggression assays suppresses the level of aggressiveness. Similar to that of wild-type flies, group housing greatly decreased the level of aggressiveness of nina $B^{1}$ mutants (Figure $1 C$ ). This result suggests that visual perception does not contribute significantly to social suppression of fly aggressiveness.

\section{Complete loss of eye does not prevent social suppression of aggression}

To further confirm above result, we also examined if complete loss of eye in the eyes absent gene (eya) mutants affects social suppression of aggression. Mutations in the eya gene cause defects in eye development [14], leading to loss of eye (Figure 2B). Like that of wild-type flies, we found that the level of aggressiveness of eya mutants was greatly suppressed by social experience prior to aggression assays (Figure 2C). This result, together with the result from testing ninaB ${ }^{1}$ mutants (Figure $1 C$ ), argue against a major role for visual perception in mediating social suppression of fly aggressiveness.
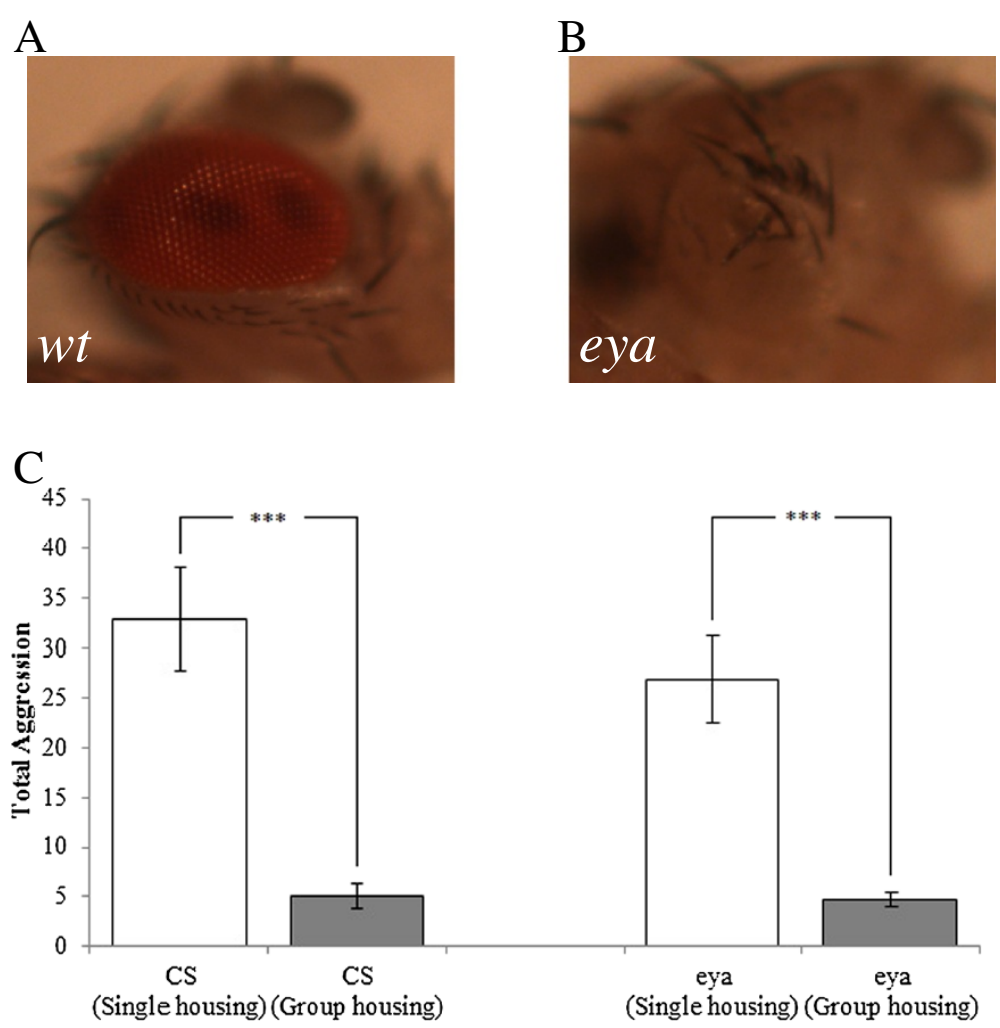

Figure 2 Complete loss of eye does not affect social suppression of aggressiveness. (A) The compound eye consists of $\sim 800$ ommatidia in wild type. (B) In eya $a^{2}$ mutants, the eye is completely absent. (C) Complete loss of eye in eya ${ }^{2}$ mutants did not affect social suppression of aggressiveness. ${ }^{* * *} \mathrm{p}<0.0001$. Pairs of flies tested: CS, $\mathrm{n}=27$ (single housing), $\mathrm{n}=21$ (group housing); eya ${ }^{2}$ mutants, $\mathrm{n}=20$ (single housing), $n=20$ (group housing). Error bars represent SEM. 
Chronic visual loss does not affect aggressiveness of single-housed flies lacking social experience prior to behavioral assays

When we examined the effects of chronic visual loss on social suppression of aggression, we found that singlehoused flies in which vision is impaired still showed high levels of aggressiveness (Figures 1 and 2). Such results are in marked contrast to a previous report that suggests that visual impairment greatly decreases aggressiveness of single-housed flies, based on analysis of white-eyed flies carrying mutations in the $w$ gene [10]. To further test the potential role of visual perception in regulating aggressiveness of single-housed flies, we performed more detailed analysis of flies with chronic visual loss.
The level of aggressiveness of isolated ninaB mutant flies was compared to that of wild-type or rescue flies in which vision was restored in nina $B$ mutants by eye-specific expression of a ninaB transgene. No significant difference in the levels of aggressiveness was observed between blind flies (i.e. ninaB mutants) and flies with normal vision (i.e. wild-type or rescue flies) (Figure 3A). Similar results were observed when the level of aggressiveness of single-housed eya flies in which the eye is absent was compared to that of wild-type or eya heterozygous flies with intact eye (Figure 3B). These results confirm that chronic visual loss does not affect the levels of aggressiveness of single-housed flies.
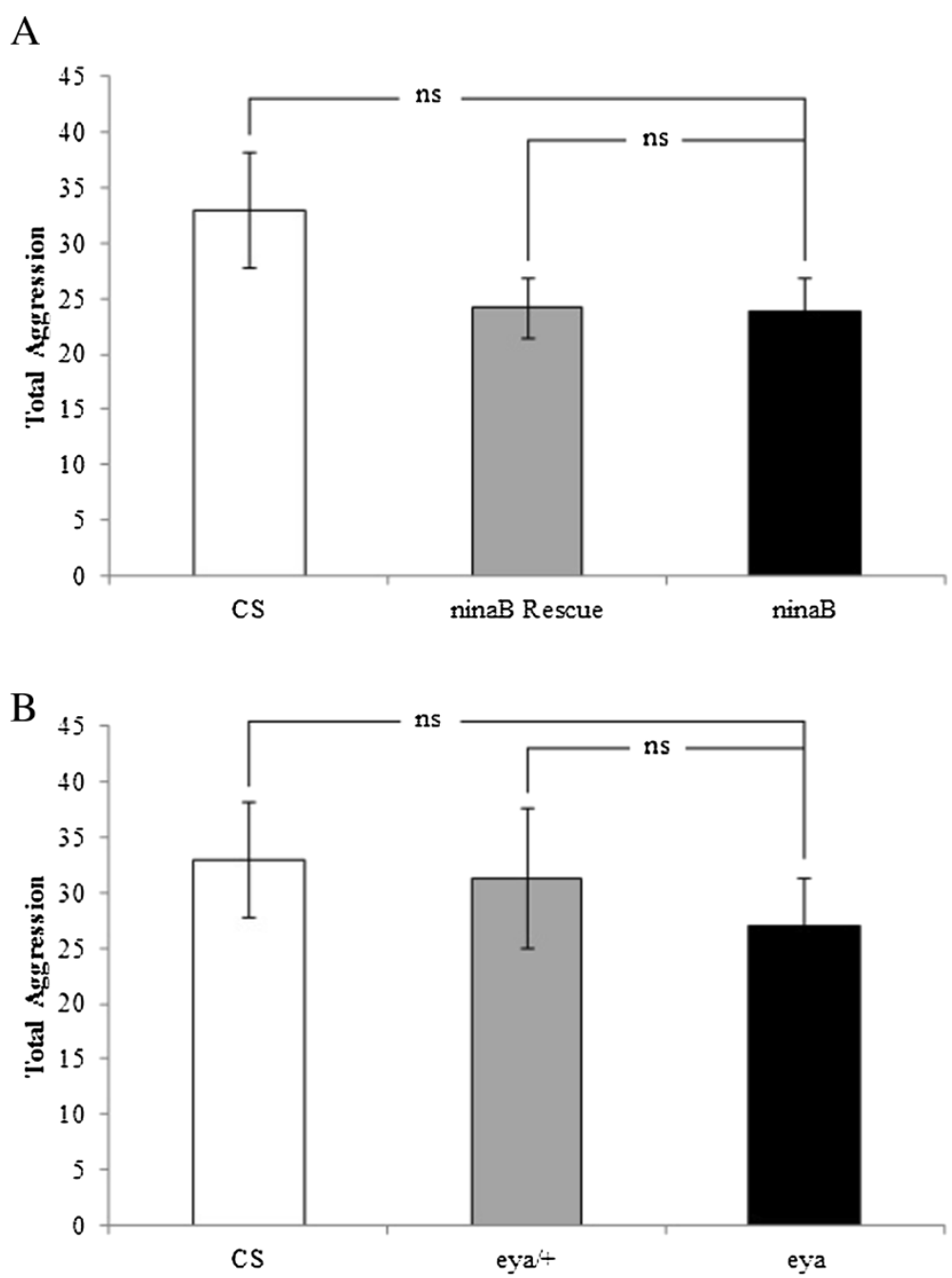

Figure 3 Chronic visual loss does not affect aggressiveness of single-housed flies that lack social experience prior to behavioral tests. (A) The level of aggressiveness (i.e. total aggression) was quantified by counting the number of all aggressive events (i.e. lunges, wing threats, tussles, boxing, and holding) within 10-min period. No significant difference was observed between single-housed wild type and ninaB ${ }^{1}$ mutants, or between single-housed nina $B^{1}$ mutants and rescued individuals in which vision was restored by eye-specific expression of a ninaB transgene in nin $a B^{1}$ mutants. Pairs of flies tested: $C S, n=27$; nina $B^{1}$ Rescue, $n=26 ; n i n a B^{1}, n=22$. (B) Loss of eye in ey $a^{2}$ mutants did not affect aggressiveness of single-housed flies. The level of aggressiveness of single-housed ey $a^{2}$ mutants was comparable to that of wild-type or ey ${ }^{2}$ heterozygous flies. Pairs of flies tested: $C S, n=27$; eya $a^{2} /+, n=20 ;$ eya $a^{2}, n=20$. ns, not significant $(p>0.05)$. Error bars represent SEM. 
To determine if chronic visual loss affects locomotor activity, we examined travel distance of wild-type, ninaB mutant or rescued flies within 10-minute period. No significant difference in travel distance was observed (Figure 4A). We also examined travel distance of eya heterozygous and homozygous flies. While loss of vision in eya homozygous mutants does not affect aggressiveness of isolated flies (Figure 3B), the locomotor activity of eya mutants was lower than that of wild-type or eya heterozygous mutants (Figure 4B).

\section{Acute blockade of visual circuit activity increases aggressiveness of single-housed flies}

We then examined if temporal blockade of visual circuit activity during the period of aggression assays affects aggressiveness of flies that were single-housed prior to behavioral assay. To test this, synaptic transmission from photoreceptor cells was temporally blocked by eyespecific expression of a temperature-sensitive form of shibire $\left(s h i^{\text {ts }}\right)$ that encodes the fly homolog of dynamin. This allows the blockade of synaptic transmission in photoreceptor cells at restrictive temperature $[15,16]$.

A shift from permissive temperature (i.e. $22^{\circ} \mathrm{C}$ ) to restrictive temperature (i.e. $32^{\circ} \mathrm{C}$ ) effectively blocked visual circuit activity, leading to loss of vision at restrictive temperature (Figure 5A). Blockade of visual circuit activity, however, did not affect locomotor activity (Figure 5B). We then examined the effects of temporally blocking visual circuit activity on the level of aggressiveness. Compared to that of flies at permissive temperature, the level of aggressiveness of single-housed flies at restrictive temperature increased significantly (Figure 5C). This
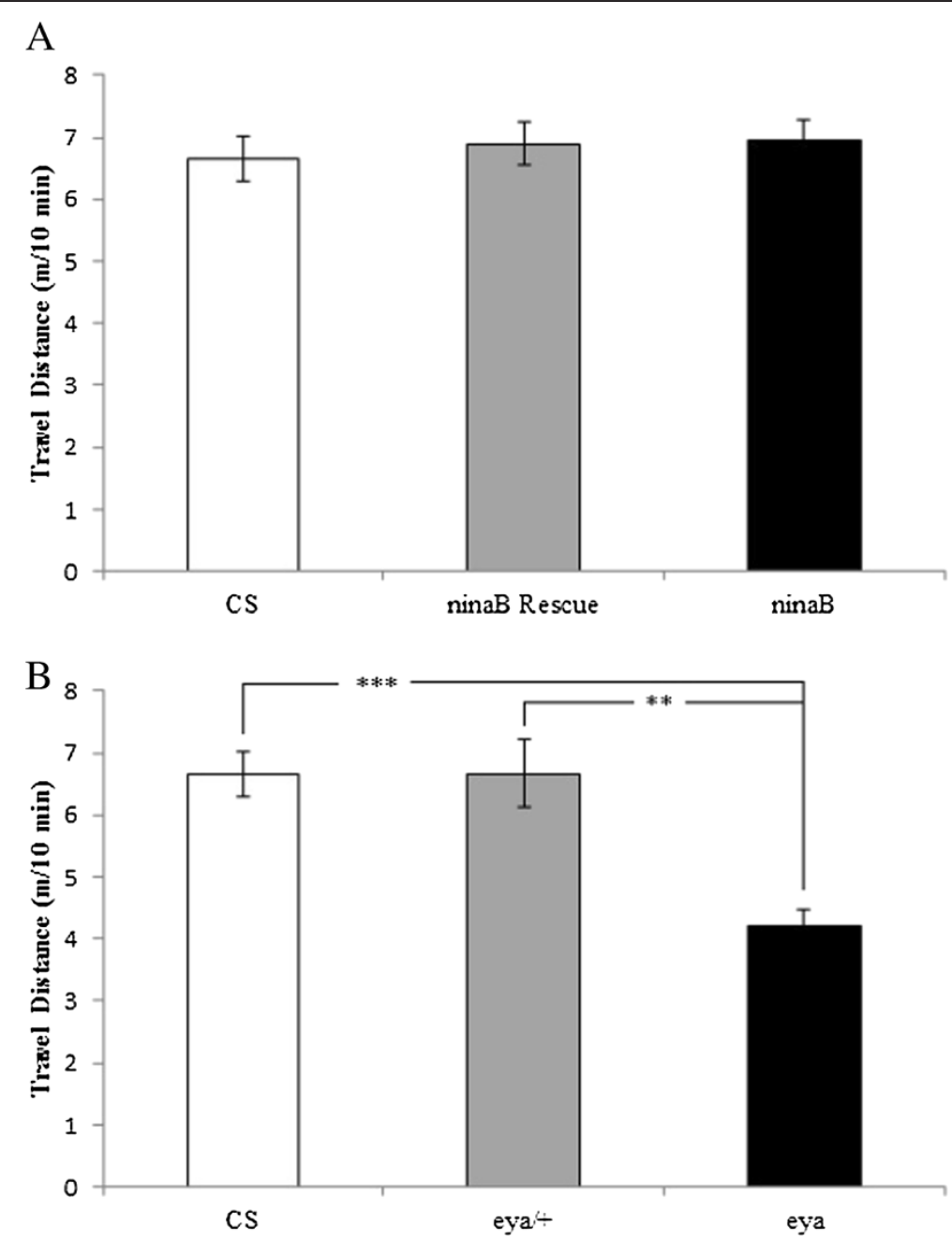

Figure 4 The effects of chronic visual impairment on fly locomotor activity. (A) Travel distance of flies within 10-min period was measured. No significant difference in locomotion was observed between ninaB ${ }^{1}$ and wild-type flies or between nina $B^{1}$ and rescued flies. Pairs of flies tested: $C S, n=30 ;$ ninaB $B^{1}$ Rescue, $n=22 ; n i n a B^{1}, n=30$. (B) Travel distance of eya ${ }^{2}$ homozygous mutant flies was measured. Compared to that of wild-type or eya ${ }^{2}$ heterozygous flies, lower locomotor activity was observed in eya ${ }^{2}$ homozygous mutant flies. Pairs of flies tested: $C S, n=30$; eya $a^{2} /+, \mathrm{n}=29 ;$ eya $^{2}, \mathrm{n}=26 .{ }^{* *} \mathrm{p}<0.01,{ }^{* * *} \mathrm{p}<0.001$. Error bars represent SEM. 

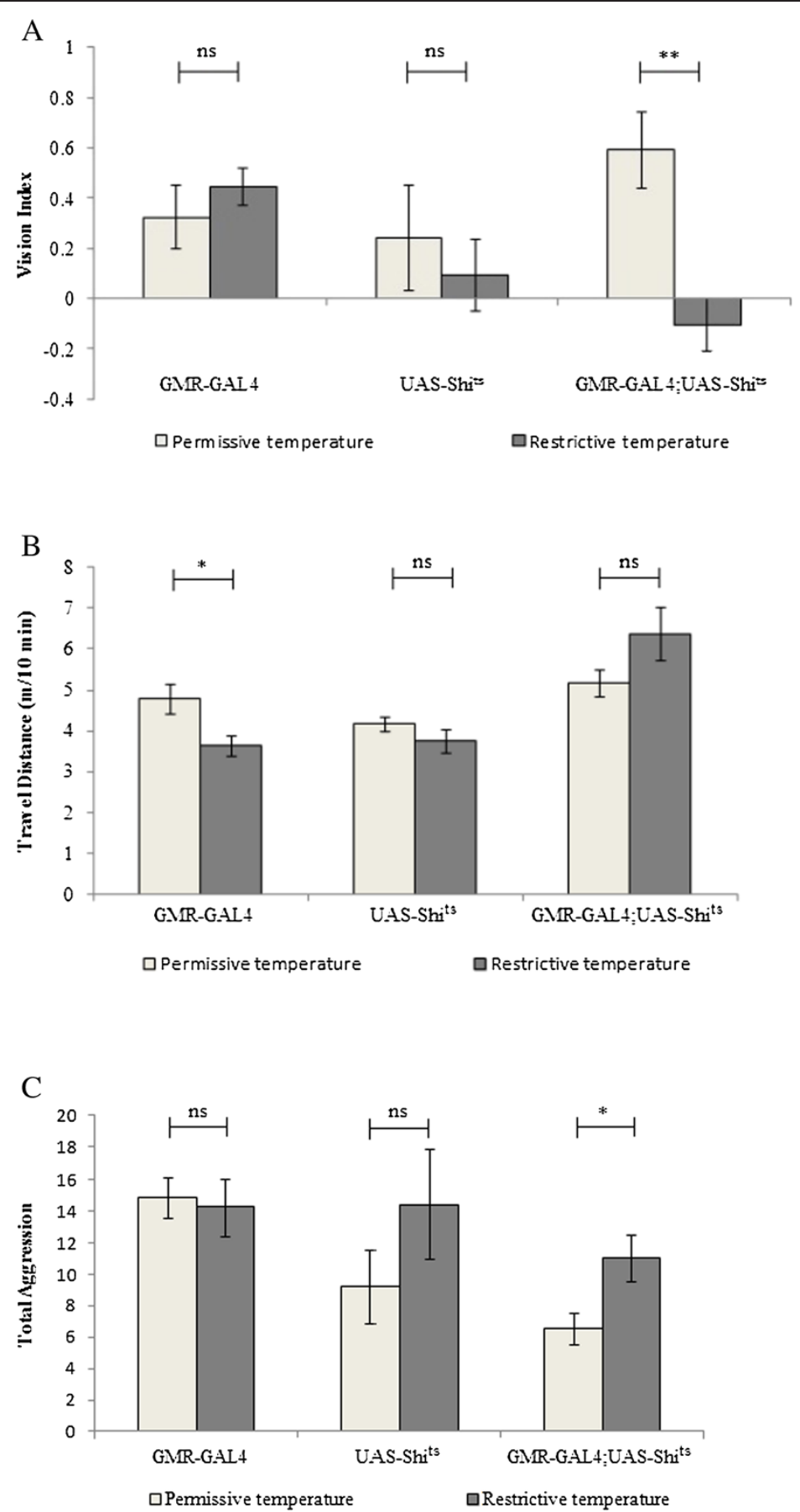

Figure 5 (See legend on next page.) 
(See figure on previous page.)

Figure 5 Temporal blockade of visual circuit activity increases aggressiveness of single-housed flies. UAS-Shits were expressed under control of eye-specific driver GMR-GAL4, which blocks synaptic transmission in photoreceptor cells at restrictive temperature $\left(32^{\circ} \mathrm{C}\right)$. Flies that only carry GMR-GAL4 or UAS-Shits were used as controls. Vision index (A), locomotor activity (B) and aggression (C) of flies were examined. The performance at restrictive temperature $\left(32^{\circ} \mathrm{C}\right)$ was compared to that of same-genotype flies at permissive temperature $\left(22^{\circ} \mathrm{C}\right)$. (A) Blockade of photoreceptor synaptic transmission impaired fly vision. ${ }^{* *} p<0.01$. "ns" indicates $p>0.05$. Number of experiments performed at permissive temperature: GMR-GAL4, $n=9$; UAS-Shits, $n=10 ; G M R-G A L 4 ; U A S-S h i^{\text {ts }}, n=9$. Number of experiments performed at restrictive temperature: GMR-GAL4, $n=10 ;$ UAS-Shi $^{\text {ts }}, \mathrm{n}=8 ;$;MR-GAL4;UAS-Shit, $\mathrm{n}=9$. (B) Travel distance of flies within 10-min period was measured. ${ }^{\text {* }} \mathrm{p}<0.05$. Pairs of

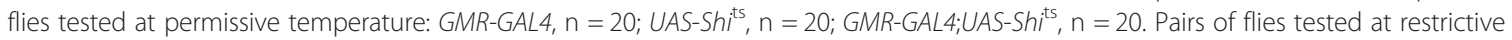
temperature: GMR-GAL4, $n=20$; UAS-Shit,$n=21 ; G M R-G A L 4 ; U A S-S h i^{\text {ts }}, n=19$. (C) Aggressiveness of single-housed flies in which photoreceptor synaptic transmission was temporally blocked at restrictive temperature was compared to that of flies at permissive temperature. ${ }^{*} p<0.05$. Pairs of flies tested at permissive temperature: GMR-GAL4, $n=20 ;$ UAS-Shits $n=20 ; G M R-G A L 4 ; U A S-S h i i^{\text {ts }}, \mathrm{n}=20$. Pairs of flies tested at restrictive temperature: GMR-GAL4, $\mathrm{n}=20 ;$ UAS-Shit $^{\text {ts }}, \mathrm{n}=21$; GMR-GAL4;UAS-Shits, $\mathrm{n}=19$. Error bars represent SEM.

result suggests that visual perception helps decrease aggressiveness of single-housed flies.

\section{Discussion}

Social suppression of aggression is observed in both vertebrates and invertebrates. While pheromonal activation of olfactory neurons has been implicated in this process in Drosophila, it remains unclear if other sensory cues also contribute to the modulation of aggressiveness by social experience. In this study, we investigated the effects of manipulating visual circuit activity in the control of fly aggression. We showed that blockade of visual circuit activity does not prevent social suppression of aggression. While chronic blockade of visual circuit activity does not affect aggressiveness of single-housed flies that lack social experience prior to behavioral tests, acute blockade of visual circuit activity increases the level of aggressiveness of single-housed flies.

Our results indicate that visual perception is not a major factor that allows male flies to recognize and interact with each other for suppressing aggressiveness by social experience. Whereas pheromonal activation of certain neurons in the olfactory system has been shown to contribute significantly to social suppression of aggression [8,9]. Recent studies showed that the gustatory system also plays a role in modulating fly aggression [17-19]. Future studies are required to determine if gustatory cues contribute to social suppression of fly aggressiveness.

Our result showing that acute blockade of visual circuit activity increases the level of aggressiveness of single-housed flies is surprising. Previous work by Heisenberg and colleagues showed that mutants defective in the $w$ gene display much lower levels of aggressiveness [10]. Since the $w$ gene mediates eye pigmentation, this result suggests that visual perception promotes aggressiveness. However, since removing $w$ gene in the brain also causes a decrease in aggression [10], together with that white-eyed cricket mutants display normal levels of aggressiveness [20], we speculate that the decrease in the level of aggressiveness of white-eyed flies may not be caused by vision impairment.

While acute blockade of visual circuit activity increases the level of aggressiveness of isolated flies, chronic blockade of visual circuit activity does not affect fly aggressiveness. One possible explanation is that chronic blockade of visual circuit activity increases the sensitivity of fly response to other sensory cues, which may compensate for loss of visual perception in decreasing aggressiveness. Future studies are required to address these possibilities.

\section{Conclusion}

Visual circuit activity does not contribute significantly to social suppression of aggression in Drosophila. For individuals reared in isolation and thus lack social experience prior to behavioral tests, however, visual perception helps decrease the level of aggressiveness.

\section{Materials and methods}

\section{Stocks and rearing condition}

nina $B^{1}$ and ey $a^{2}$ mutants were obtained from Bloomington stock center. ninaB rescue experiments were performed by generating GMR-GAL4/+; ninaB ${ }^{1}$, UAS-ninaB/ ninaB ${ }^{1}, U A S$-ninaB flies. UAS-Shits were provided by Dr. Greg Suh (NYU). GMR-GAL4;UAS-Shit flies were generated by crossing male UAS-Shits with female GMR-GAL4 flies. Canton-S flies were used as wild-type controls. Flies were reared at $25^{\circ} \mathrm{C}$ with $50-60 \%$ humidity and 12 hour light-dark cycle. Newly emerged males from pupal cases were single-housed in a $2 \mathrm{ml}$ microfuge tube containing $1 \mathrm{ml}$ of fly food for 6 days prior to aggression and locomotion assays. For experiments testing social influence of fly aggressiveness, flies were grouped in vials (10 flies per vial) and reared for 6 days prior to aggression assay. For vision tests, flies were group housed (7-12 flies) per vial and reared for 5-7 days prior to vision tests.

\section{Vision assay}

To examine fly vision, we used standard vials with $1.2 \mathrm{~cm}$ radius and $9.5 \mathrm{~cm}$ height. One vial was completely covered 
with dark duct tape except for the tip where flies were aspirated, and was indicated as dark zone. Another uncovered transparent vial was indicated as light zone. The two vials were attached together, separated by a paper cardboard, and horizontally placed under a light source (Figure 1A). For each experiment, 7-12 flies were gently aspirated into either dark or light zone. Flies were allowed to get accustomed to new environment for $5 \mathrm{~min}$. The cardboard was then removed gently in a way that did not agitate the flies. Flies were let freely move between light and dark zone for $10 \mathrm{~min}$. The number of flies in light zone and dark zone was counted. Vision index is defined as: (number of light-zone flies - number of dark-zone flies)/total number of flies.

\section{Aggression assay}

Aggression assay was performed by placing a pair of male flies in a circular fighting chamber $(7 \mathrm{~mm}$ radius and $3.5 \mathrm{~mm}$ height), which has a central pad (4 mm radius) covered with food, and outer space filled with agar to reduce the dehydration of food. Behavioral tests were carried out at $22^{\circ} \mathrm{C}$. For experiments involving acute blockade of visual circuit activity, aggression tests were performed at $22^{\circ} \mathrm{C}$ (permissive temperature) or $32^{\circ} \mathrm{C}$ (restrictive temperature). Two male flies of the same genotype were gently aspirated to the fighting chamber. After 5 minutes for flies to get accustomed to the environment, their behaviors were recorded with a high definition (HD) camera under fluorescent lamp for 10 minutes. The total number of aggressive events (i.e. lunges, wing threats, tussles, boxing, and holding) per 10-min period was used to indicate the level of aggressiveness.

\section{Locomotion assay}

Movement of two flies in a small round chamber was videotaped and analyzed by CADABRA software [21]. Two flies were gently aspirated into a chamber similar to the fighting chamber used for aggression assay. Movement of flies was recorded for $10 \mathrm{~min}$.

\section{Statistical analysis}

Data were expressed as mean \pm SEM and processed by commercially available GraphPad Prism ${ }^{\circ}$ 5.0. Mann Whitney test, or Kruskal-Walis ANOVA test followed by Dunn's multiple comparison test were used in statistical analysis. $\mathrm{p}$-value less than $0.05(\mathrm{p}<0.05)$ is considered as significant.

\section{Competing interests}

The authors declare that they have no competing interests.

\section{Authors' contributions}

MR conducted the experiments, and was involved in writing the manuscript. CD and DS helped data quantitation. YR supervised the project and wrote the manuscript. All authors read and approve the manuscript.

\section{Acknowledgements}

We thank members of the Rao laboratory for comments and discussions; the Bloomington Stock Center for mutant lines; Dr. Greg Suh for UAS-shis ${ }^{\text {ts }}$. This work was supported by an operating grant (Rgpin 341431-10) from Natural Science and Engineer Research Council of Canada, and a China-Canada Joint Health Research Initiative Grant (FRN109611) awarded to Yong Rao.

\section{Author details}

${ }^{1}$ McGill Centre for Research in Neuroscience, McGill University Health Centre, 1650 Cedar Avenue, Montreal, Quebec H3G 1A4, Canada. ${ }^{2}$ Department of Neurology and Neurosurgery, Integrated Program in Neuroscience, McGill University Health Centre, 1650 Cedar Avenue, Montreal, Quebec H3G 1A4, Canada. ${ }^{3}$ Department of Medicine, McGill University Health Centre, 1650 Cedar Avenue, Montreal, Quebec H3G 1A4, Canada.

Received: 12 June 2014 Accepted: 24 July 2014

Published: 13 August 2014

\section{References}

1. Anholt RR, Mackay TF: Genetics of aggression. Annu Rev Genet 2012, 46:145-164.

2. Loeber R, Hay D: Key issues in the development of aggression and violence from childhood to early adulthood. Annu Rev Psychol 1997, 48:371-410

3. Matsumoto K, Pinna G, Puia G, Guidotti A, Costa E: Social isolation stress-induced aggression in mice: a model to study the pharmacology of neurosteroidogenesis. Stress 2005, 8:85-93.

4. Luciano D, Lore R: Aggression and social experience in domesticated rats. J Comp Physiol Psychol 1975, 88:917-923.

5. Ferno A: The effect of social isolation on the aggressive and sexual behaviour in a cichlid fish, Haplochromis burtoni. Behaviour 1978, 65:43-61.

6. Hoffman AA: The influence of age and experience with conspecifics on territorial behavior inDrosophila melanogaster. J Insect Behav 1990, 3:1-12.

7. Wang L, Dankert H, Perona P, Anderson DJ: A common genetic target for environmental and heritable influences on aggressiveness in Drosophila. Proc Natl Acad Sci U S A 2008, 105:5657-5663.

8. Wang $L$, Anderson DJ: Identification of an aggression-promoting pheromone and its receptor neurons in Drosophila. Nature 2010, 463:227-231.

9. Liu W, Liang X, Gong J, Yang Z, Zhang YH, Zhang JX, Rao Y: Social regulation of aggression by pheromonal activation of Or65a olfactory neurons in Drosophila. Nat Neurosci 2011, 14:896-902.

10. Hoyer SC, Eckart A, Herrel A, Zars T, Fischer SA, Hardie SL, Heisenberg M: Octopamine in male aggression of Drosophila. Curr Biol 2008, 18:159-167.

11. von Lintig J, Dreher A, Kiefer C, Wernet MF, Vogt K: Analysis of the blind Drosophila mutant ninaB identifies the gene encoding the key enzyme for vitamin A formation invivo. Proc Natl Acad Sci U S A 2001, 98:1130-1135.

12. Stephenson RS, OTousa J, Scavarda NJ, Randall LL, Pak WL: Drosophila mutants with reduced rhodopsin content. Symp Soc Exp Biol 1983, 36:477-501.

13. Hotta $Y$, Benzer $S$ : Genetic dissection of the Drosophila nervous system by means of mosaics. Proc Natl Acad Sci U S A 1970, 67:1156-1163.

14. Bonini NM, Leiserson WM, Benzer S: Multiple roles of the eyes absent gene in Drosophila. Dev Biol 1998, 196:42-57.

15. Kitamoto T: Conditional modification of behavior in Drosophila by targeted expression of a temperature-sensitive shibire allele in defined neurons. J Neurobiol 2001, 47:81-92.

16. Zhou Y, Cameron S, Chang WT, Rao Y: Control of directional change after mechanical stimulation in Drosophila. Mol Brain 2012, 5:39.

17. Fernandez MP, Chan YB, Yew JY, Billeter JC, Dreisewerd K, Levine JD, Kravitz EA: Pheromonal and behavioral cues trigger male-to-female aggression in Drosophila. PLOS Biol 2010, 8:e1000541.

18. Miyamoto T, Amrein H: Suppression of male courtship by a Drosophila pheromone receptor. Nat Neurosci 2008, 11:874-876.

19. Wang L, Han X, Mehren J, Hiroi M, Billeter JC, Miyamoto T, Amrein H, Levine $J D$, Anderson DJ: Hierarchical chemosensory regulation of male-male social interactions in Drosophila. Nat Neurosci 2011, 14:757-762. 
20. Sakura M, Watanabe T, Aonuma H: Aggressive behavior of the white-eye mutant crickets, Gryllus bimaculatus. Acta Biol Hung 2012, 63(Suppl 2):69-74.

21. Dankert H, Wang L, Hoopfer ED, Anderson DJ, Perona P: Automated monitoring and analysis of social behavior in Drosophila. Nat Methods 2009, 6:297-303.

doi:10.1186/s13041-014-0055-0

Cite this article as: Ramin et al: Aggression and social experience:

genetic analysis of visual circuit activity in the control of aggressiveness in Drosophila. Molecular Brain 2014 7:55.

\section{Submit your next manuscript to BioMed Central and take full advantage of:}

- Convenient online submission

- Thorough peer review

- No space constraints or color figure charges

- Immediate publication on acceptance

- Inclusion in PubMed, CAS, Scopus and Google Scholar

- Research which is freely available for redistribution 